\title{
Initial Experience of Transesophageal Echocardiography in NAMS Bir Hospital
}

Shah RK*

*Department of Cardiology, NAMS, Bir Hospital, Kathmandu, Nepal

\section{ABSTRACT}

A retrospective study of patients undergoing trans esophageal echocardiogram was conducted in Bir Hospital, from April 2008 to March 2010. A total of 51 patients were included in the study. Among the 51 patients 29 (56.8\%) were female and 22 (43.2\%) were male. Mean age was $38+/-$ 15.1 (range 12-77 years). The improved resolution and anatomic detail provided by TEE makes it such a powerful diagnostic tool. TEE is a safe outpatient procedure. Viscous two percent lidocaine topical anesthesia was adequate for the procedure. Common indication for TEE was to confirm Atrial septal defect and Left atrial clot. Complications of TEE were very few. In this study most common problems were nausea and vomiting in 22 cases (43.1\%) during procedure. Sore throat was in 3 cases $(5.8 \%)$ after the procedure. Ventricular premature contractions were seen in I case $(1.9 \%)$.

Key words: Transesophageal echocardiography, Transthoracic echocardiography

Correspondence:

Dr Ram Kishor Shah

NAMS, Bir Hospital

Post Box: 8011

Kathmandu,Nepal

Tel: 00977-1-4221119, 4221988

Fax: 00977-1-4247032

Email: rkshah40@yahoo.com 


\section{INTRODUCTION}

In 1976, Frazin and colleagues ${ }^{1}$ first attached a single crystal M-mode ultrasound transducer to the tip of a cable for esophageal imaging. Since then, improvements in ultrasound technology coupled with miniaturization of transducers and the development of soft flexible tubing have led to a virtual exponential growth of transesophageal echocardiography (TEE). Multiplane transducer technology is the latest innovation in the rapidly changing field of TEE. It consists of a single array of crystals that is electronically or mechanically rotated about the long axis of the sound beam in a $180^{\circ}$ arc, producing a circular continuum of tomographic, two-dimensional transverse and longitudinal images ${ }^{2,3}$. The primary advantage of multiplane technology is the ease with which an uninterrupted series of adjacent images can be obtained with minimal needs for repositioning of the probe tip. Thus, after nearly a decade of multicentre experience and rapidly advancing probe technology, TEE has truly become a major part of the diagnostic and "therapeutic" armamentarium of a cardiologist.

\section{MATERIALS AND METHODS}

A retrospective study that was conducted in the department of cardiology of NAMS, Bir Hospital, Kathmandu from April 2008 to March 2010 AD. All patients with indications for the procedure were subjected to TEE. The instrument used was Toshiba Xario (PST 50 AT, $5 \mathrm{MHZ}$ ). The procedure was explained to the patient, and an informed consent was obtained from the subjects. All data were analyzed by using statistical package for social science (SPSS) version 10 for windows.

\section{INDICATIONS}

1. As an adjunct to transthoracic echocardiography (TTE) when the quality of the latter is unsatisfactory4 (i.e. large patient, presence of a prosthetic valve, thoracic deformities, etc.)

2. When the transthoracic window to the heart is impeded or unobtainable (i.e. in the operating room, catheterization laboratory and intensive care unit, etc.)

3. For intraoperative evaluation, particularly when significant residual cardiac abnor- malities (i.e. outflow tract obstruction, valve regurgitation or stenosis, intracardiac communication) are anticipated or suspected.

4. For intraoperative monitoring of ventricular function in patients with congenital heart disease undergoing non-cardiac surgery.

5. For guidance of interventional procedures during cardiac catheterization (i.e. balloon interventions, placement of transcatheter devices, radiofrequency ablation, etc.)

6. When vegetations or masses (which are poorly-imaged from transthoracic windows) are suspected to be present.

\section{CONTRAINDICATIONS}

TEE is an invasive procedure; thus, when the risk to patient health outweighs the benefit of the procedure, a contraindication (relative or absolute) exits $^{4,5}$.

\section{Absolute contraindications:}

1. Esophageal obstruction or stricture

2. Active gastrointestinal bleeding

3. Perforated viscus

4. Unrepaired tracheoesophageal fistula

5. Severe respiratory decompensation

6. Inadequate control of the airway

\section{Relative contraindications:}

1. Esophageal varices

2. Esophageal diverticulum

3. Cervical spine injury or deformity

4. Oropharyngeal distortion or deformity

5. Post esophageal surgery

6. Severe coagulopathy

\section{Preparation :}

A careful history was obtained before performing the transesophageal echocardiographic examination. In addition to a thorough cardiovascular history, specific questions was asked regarding past esophageal injury or surgery, swallowing difficulty, gastrointestinal bleeding, medications (e.g., aspirin), and allergies to medications (e.g. lidocaine). To minimize the risk of aspiration during the procedure the patient had nothing by mouth for at least four hours and preferably six to eight hours before the procedure. The procedure was explained to the patient, and an informed consent was obtained ${ }^{6}$. Topical anesthesia consisting of viscous lidocaine gargle was routinely used during the procedure. The Patient was advised not to 
take anything by mouth for 30 to 60 minutes after the procedure ${ }^{4}$. The incidence of bacteremia $(4 \%$ to $8 \%$ ) and the risk of endocarditis with upper endoscopy are considered negligible in most published reports ${ }^{7-11}$. In the recent recommendations of the American heart Association ${ }^{12}$, endoscopy with or without gastrointestinal biopsy is considered a low risk procedure in which endocarditic prophylaxis is not recommended.

\section{RESULTS}

A total of 51 patients were included in the study. Among the 51 patients $29(56.8 \%)$ were female and $22(43.2 \%)$ were male. Mean age was $38+/-15.1$ (range 12-77 years) and majority of them had atrial septal defect. (Table-1)

Table -1

$\begin{array}{lcc}\text { Diseases } & \text { Frequency } & \text { Percent } \\ \text { Atrial septal defect (ASD) } & 25 & 49.0 \\ \text { Constrictive Pericarditis (CP) } & 4 & 7.8 \\ \text { Left atrial clot (LA clot) } & 4 & 7.8 \\ \text { Normal } & 11 & 21.6 \\ \text { Pericardial cyst } & 1 & 2.0 \\ \text { Prosthetic valve } & 2 & 3.9 \\ \text { Right atrial Myxoma } & & \\ \text { (RA Myxoma) } & 3 & 5.9 \\ \text { Vegetation(s) } & 1 & 2.0 \\ \text { Total } & 51 & 100\end{array}$

Transesophageal Echocardiographic assessment of heart diseases.

\section{DISCUSSION}

In general TEE is performed when the information obtained by TTE is insufficient and to obtain better images when transthoracic images are inadequate. The proximity of the esophagus to the heart allows for improved visualization of many cardiac structures, particularity those that are posteriorly located ${ }^{13}$. In reported series, the incidence of major and minor complications is $2 \%$ to $3 \%$ with most being minor complications ${ }^{13,14}$. Ma- jor complications (death, esophageal perforation, significant arrhythmias, congestive heart failure, and aspiration) occur with a frequency of $0.3 \%$, with a reported mortality of less than $0.01 \%$. Reported minor complications include transient hypotension, hypertension (particularly with agitation), hypoxia, and arrhythmias (such as sustained ventricular tachycardia, nonsustained ventricular tachycardia, and transient atrioventricular block). Methemoglobinemia has been rarely reported due to the anesthetic spray and should be considered if cyanosis occurs. In this study, the most common problems encountered were nausea and vomiting which was seen in 22 cases $(43.1 \%)$ during the procedure. Sore throat was noted in 3 cases (5.8\%) after the procedure. Ventricular premature contractions were seen in one case $(1.9 \%)$. The improved resolution and anatomic detail provided by TEE, as compared with TTE is what makes it such a powerful diagnostic tool. However, this can also lead to misinterpretation of normal structures, trabeculations in the atrial appendage can be mistaken for thrombi, and lipomatous hypertrophy of the inter-atrial septum may be incorrectly labeled as a mass, as can the Eustachian valve, The transverse and oblique sinuses can be mistaken for abscess cavities ${ }^{15}$. These pitfalls are best minimized by the experience of the operator, but variations in anatomy may provide diagnostic dilemmas for even the most skilled echocardiographer.

\section{CONCLUSION}

TEE is a safe outpatient procedure. Viscous two percent lidocaine topical anesthesia was adequate for the procedure. Common indication for TEE was to confirm the presence of ASD and to detect LA clot. Complications of TEE were very few. Misinterpretations of normal structures are best minimized by the experience of the operator.

\section{ACKNOWLEDGEMENT}

I would like to express my sincere thanks to sister Durga, Srijana, Laxmi, Anita, Rajaram, Sarita and other staffs of cardiac unit, who were directly or indirectly involved in this study. 


\section{REFRERENCES}

1. Frazin L et al: Esophageal echocardiography, Cirulation 54:102, 1976.

2. Seward JB et al: Multiplane transesophageal echocardiography: image orientation, examination technique, anatomic correlations, and clinical applications, Mayo Clin Proc 68:523, 1993.

3. Pandian NG et al: Multiplane transesophageal echocardiography, Echocardiography 9:649, 1992.

4. Fleischer DE, Goldstein SA: Transesophageal echocardiography: what the gastroenterologist thicks the cardiologist should know about endoscopy, J Am Soc Echocardiogr 3:428, 1990.

5. Ament ME: Fiberoptic upper intestinal endoscopy in infants and children, pediatr Clin North Am 35:141, 1988.

6. Fleischer DE: Monitoring the patient receiving conscious sedation for gastrointestinal endoscopy: issues and guidelines, Gastrointest Endosc 35:262, 1989.

7. Botoman VA, Surawicz CM: Becteremia with gastrointestinal endoscopic procedures, Gastrointest Endosc 32:342, 1986.

8. Leitch DG et al: Bacteremia following endoscopy, Br J Clin Pract 40:341, 1986.

9. Norfleet RG et al: Does bacteremia follow upper gastrointestinal endoscopy? Am J Gastroenterol 76:420, 1981.
10. Perucca PJ, Meyer GW: Who should have endocarditis prophylaxis for upper gastrointestinal procedures? Gastrointest Endosc 31:285, 1985.

11. Shorvon PJ, Ey Kyn SJ, Cotton PB: Gastrointestinal instrumentation, bacteremia, and endocarditis, Gut 24:1078, 1983.

12. DaJani AS et al: Prevention of bacterial endocarditis: a statement from the committee on Rheumatic Fever, Endocarditis, and Kawasaki Disease of the Council on Cardiovascular Disease in the young of the American Heart Association, JAm Med Assoc 264:2919, 1990.

13. Cheitlin MD, et al. ACC/AHA/ASE 2003 Guideline update for the Clinical Application of Echocardiography: summary article. A report of the American College of Cardiology/American Heart Association Task Force on Practice Guidelines. J Am coll cardiol 2003;42;954-970.

14. Douglas PS, et al. ACCF/ASE/ACEP/ASNC/ SCAI/SCCT/SCMR 2007 appropriateness criteria for transthoracic and transesophageal echocardiography. J Am call cardio 2007;50;187-204.

15. Khanderia BK, Seward JB Tajik AJ. Critical appraisal of transesophageal echocardiography: limitations and pitfalls. Crit care clin 1996;12:235-251. 